\title{
Rôle des champignons mycorhiziens à arbuscules de zones arides dans la résistance du trèfle (Trifolium alexandrinum L.) au déficit hydrique
}

\author{
Abdelilah MedDiCH ${ }^{\mathrm{a}}$, Abdallah OIHABI $^{\mathrm{a} *}$, Younes ABBAs ${ }^{\mathrm{a}}$, Essia BIZID $^{\mathrm{b}}$ \\ ${ }^{a}$ Laboratoire de Physiologie Végétale, BP S/15, Faculté des Sciences-Semlalia, Marrakech, Maroc \\ ${ }^{\mathrm{b}}$ Laboratoire de Physiologie Végétale, Campus universitaire 1060, Faculté des Sciences de Tunis, Tunisie
}

(Reçu le 30 juin 1999; révisé le 18 janvier 2000; accepté le 3 février 2000)

\begin{abstract}
Résumé - La tolérance du trèfle mycorhizé (Trifolium alexandrinum L.) à la sécheresse diffère selon les isolats de champignons mycorhiziens associés à la plante hôte. Cinq isolats fongiques ont été isolés à partir des sols de palmeraies marocaines et étudiés pour leur aptitude à améliorer la tolérance du trèfle au stress hydrique. L'application d'une contrainte hydrique de $30 \%$ de la capacité au champ réduit sévèrement la progression des champignons mycorhiziens dans le cortex racinaire du trèfle. Les isolats provenant de la palmeraie d'Aoufous sont moins affectés par le dessèchement du sol que les Glomus et Sclerocystis d'Agdz. L'effet de la mycorhization quant à l'amélioration de la production de biomasse du trèfle est bien apparu dans le cas d'infection par les isolats fongiques d'Aoufous et le Glomus mosseae (souche de référence provenant de l'INRA de Dijon, France). Ces mêmes champignons mycorhiziens ont permis au trèfle de maintenir sa teneur en eau, son potentiel hydrique et sa transpiration foliaire à des valeurs élevées par rapport au témoin non inoculé. Les valeurs de la résistance des stomates et du déficit de saturation en eau sont restées plus faibles chez les plantes mycorhizées que chez les plantes non mycorhizées. On note également, que les isolats du complexe d'Aoufous et le Glomus mosseae se sont montrés les plus agressifs dans la colonisation des racines du trèfle et ce quel que soit le niveau du traitement hydrique imposé au sol. Les isolats fongiques provenant des sols de la palmeraie d'Aoufous se sont montrés aussi efficaces que Glomus mosseae.
\end{abstract}

mycorhize / croissance / relation hydrique / sécheresse / zone aride

Abstract - Effect of arbuscular mycorrhizal fungi on drought resistance of clover. Tolerance of mycorrhized clover (Trifolium alexandrinum L.) to drought depends on the arbuscular mycorrhizal fungi associated to the host plant. Five arbuscular mycorrhizal fungi isolates were collected from five Moroccan date palm grove's soils and were investigated for their ability to improve the plant tolerance to water deficit stress. Applying a constraint of $30 \%$ field capacity reduces severely the mycorrhizal rate in the root cortex of most of the tested isolates. Fungi collected from Aoufous

Communiqué par Gérard Guyot (Avignon, France)

* Correspondance et tirés-à-part

oihabi@ucam.ac.ma 
date palm grove were less affected by the drought than the other strains, such as Glomus and Sclerocystis isolated from Agdz soil. The effect of mycorrhization on the biomass production occurred for the isolate of Aoufous and Glomus mosseae (reference strain obtained from INRA Dijon, France). These fungi allowed the plants to maintain its water content, water potential and its leave transpiration at high levels compared to the non inoculated plants. The values of stomata resistance and the saturation deficit in water remained lower for mycorrhized plants than non-mycorrhized. Ones further more, the isolate of Aoufous and Glomus mosseae were the most virulent colonizing the host plants regard less of the level of water deficit in the soil. Autochthon isolates originating Aoufous date palm grove was as efficient as Glomus mosseae.

mycorrhiza / growth / water deficit / drought / and arid area

\section{Introduction}

Durant la dernière décennie, la sécheresse et la salinité ont causé de sérieux préjudices dans les régions arides rendant toute activité agricole difficile voire impossible. Cette situation est à l'origine de nombreux problèmes socio-économiques graves. En effet, le déficit hydrique accompagné de montée de sels limitent considérablement les rendements agricoles dans les sols de palmeraies marocaines, ce qui pousse les populations locales à l'exode rural vers les centres urbains. D'où la nécessité de chercher des méthodes et des pratiques culturales qui viseraient à développer, chez les plantes cultivées dans ces régions, des mécanismes de résistance aux stress hydrique et salin, et par voie de conséquence, contribueraient à la restauration d'une activité agronomique adéquate permettant la stabilisation des populations sahariennes. La sélection de champignons mycorhiziens pour leur aptitude à améliorer la croissance et les relations hydriques des plantes soumises en permanence aux stress abiotiques pourrait constituer une stratégie capitale permettant la restauration et la réalisation d'une agriculture convenable dans les palmeraies marocaines comme cela a été vérifié dans des conditions similaires [42]. L'amélioration des relations hydriques des plantes mycorhizées pourrait être attribuée à une augmentation de la nutrition phosphatée, de l'absorption d'eau [20, 22, 33] ou à une augmentation des produits de la photosynthèse (sucres et protéines) des plantes inoculées [46].

Notre travail vise la recherche de moyens biologiques susceptibles d'améliorer la tolérance des plantes des régions désertiques marocaines aux contraintes abiotiques (sécheresse et salinité). L'utilisation de champignons mycorhiziens résistants aux contraintes hydriques et capables d'aider les plantes, auxquelles ils sont associés, à tolérer les conditions climatiques défavorables $[3,6,31$, 47] est l'un des moyens biologiques prometteurs.

Dans un travail antérieur [36], nous avons sélectionné des champignons mycorhiziens à arbuscules (MA), Glomus et Sclerocystis isolés des sols de palmeraies marocaines (Aoufous et Agdz) pour leur aptitude à améliorer la croissance et la nutrition minérale du trèfle (Trifolium alexandrinum L.). Le travail que nous présentons porte sur l'effet de ces champignons MA sur la tolérance du trèfle au stress hydrique en comparaison avec Glomus mosseae afin de sélectionner les souches autochtones de champignons MA les plus efficaces.

\section{Matériel et méthodes}

\subsection{Préparation et multiplication de l'inoculum mycorhizien}

L'identification des genres de champignons mycorhiziens à partir des spores collectées des sites marocains d'Aoufous et d'Agdz, sélectionnés [36] a été réalisée selon la classification de Hall [23]. Les spores ont été collectées selon la méthode décrite par Gerdman et Nicolson [21] et conservées dans du polyvinylactoglycérol à $4{ }^{\circ} \mathrm{C}$ jusqu'à leur utilisation [17]. Les champignons MA ont ensuite été multipliés sur racines de plants d'orge (Hordeum vulgare L.) [4]. Des graines d'orge ont 
été désinfectées par immersion dans une solution d'eau oxygénée à $15 \%$ pendant 30 minutes suivie de 3 lavages à l'eau distillée stérile. Elles ont été mises en germination dans la vermiculite stérile $\left(200{ }^{\circ} \mathrm{C}, 3\right.$ heures $)$ préalablement arrosée à l'eau distillée stérile. Après une semaine de germination, les plants d'orge ont été inoculés par l'application de 50 spores de champignons mycorhiziens contre le système racinaire de chaque plante [54]. Les plantes ont ensuite été repiquées dans des pots en plastique $(13 \times 9 \mathrm{~cm})$ contenant du sable auparavant désinfecté par une attaque à l'acide chlorhydrique N/3 pendant une nuit, suivie de plusieurs lavages à l'eau distillée pour éliminer les déchets organiques, puis d'une stérilisation pendant 3 heures à l'étuve à $180{ }^{\circ} \mathrm{C}$ [32]. L'ensemble a été incubé dans une salle de culture à $24{ }^{\circ} \mathrm{C}, 70 \%$ d'humidité, 16 heures de photopériode et $200 \mu \mathrm{E} \cdot \mathrm{m}^{-2} \cdot \mathrm{s}^{-1} \mathrm{~d}$ 'intensité lumineuse. Les plants d'orge ont été arrosés régulièrement à l'eau distillée avec un apport hebdomadaire de $30 \mathrm{ml}$ de solution nutritive de Long Ashton modifiée [39]. Après 2 mois de culture, les racines d'orge mycorhizées ont été désinfectées pendant 10 minutes [45], rincées trois fois pendant 10 minutes à l'eau distillée stérile, puis découpées en fragments de 1 à 2 millimètres de long. Ces derniers constituent l'inoculum mycorhizien pour la multiplication de l'inoculum sur d'autres jeunes plants d'orge et l'inoculation du trèfle. Pour caractériser cet inoculum, nous avons utilisé la technique décrite par Trouvelot et al. [51], qui permet de juger l'état de la mycorhization et reflète les potentialités du sys-

Tableau I. Caractérisation de l'inoculum constitué de racines d'orges mycorhizées.

\begin{tabular}{lc}
\hline Traitement & Fréquence d'infection (\%) \\
\hline Glomus mosseae & $88,00 \mathrm{a}$ \\
Complexe d'Aoufous & $78,89 \mathrm{a}$ \\
Glomus d'Aoufous & $76,00 \mathrm{a}$ \\
Sclerocystis Aoufous & $63,81 \mathrm{ab}$ \\
Glomus Agdz & $64,85 \mathrm{ab}$ \\
Sclerocystis Agdz & $43,94 \mathrm{~b}$
\end{tabular}

Les valeurs suivies de la même lettre ne sont pas significativement différentes au seuil $\mathrm{P}<0,05$ (test de Newman et Keuls). tème symbiotique. Dans tous les cas et après 8 semaines de culture, les fréquences d'infection $(\mathrm{F})$ des racines d'orge sont supérieures à $60 \%$; excepté dans le cas de l'inoculation par le Sclerocystis $\operatorname{Agdz}(44 \%)$ (Tab. I).

\subsection{Procédure d'application de la contrainte hydrique}

Les cultures de trèfle ont été réalisées dans des seaux noirs en plastique de cinq litres ayant un diamètre intérieur de $16 \mathrm{~cm}$ et une hauteur de $20 \mathrm{~cm}$, munis d'un dispositif de drainage pour éliminer l'eau en excès et permettre de déterminer la capacité au champ du sol. La méthode utilisée pour l'application de la contrainte hydrique est celle décrite par Elachouri [15] et Tobar et al. [49, 50]. Soit $\mathrm{P}_{1}$ le poids du seau plein de sol sec. On arrose ensuite le sol jusqu'à saturation, puis on le laisse s'égoutter sous l'effet de la pesanteur, jusqu'à poids constant. Le sol est ainsi à la capacité au champ. Soit $\mathrm{P}_{2}$ le poids du seau après écoulement de l'eau en excès. La différence $\left(\mathrm{P}_{2}-\mathrm{P}_{1}\right)$ correspondra au volume d'eau nécessaire à l'obtention de la capacité au champ du sol utilisé (100\% CC). Pour avoir des humidités équivalentes du sol, à $75 \%$ et à $30 \%$ de la capacité au champ, nous avons ajouté aux séries de seaux renfermant du sol sec, les volumes d'eau correspondant à $0,75 \times$ $\left(\mathrm{P}_{2}-\mathrm{P}_{1}\right)$ et à $0,30 \times\left(\mathrm{P}_{2}-\mathrm{P}_{1}\right)$ respectivement. On note ensuite les poids $\mathrm{P}_{3}$ et $\mathrm{P}_{4}$ des seaux à $75 \%$ et à $30 \%$ de la capacité au champ respectivement. Au cours de l'expérience, dans les deux traitements hydriques utilisés, les seaux sont ramenés par pesées successives et addition de l'eau distillée, au poids correspondant à la contrainte hydrique imposée $(75 \%$ ou $30 \%$ de la capacité au champ). Pendant toute l'expérience, les seaux sont pesés deux fois par jour (balance type Ohaus DS4 capacity $44 \times 02 \mathrm{Ib}$ ) et l'eau perdue est remplacée [50].

\subsection{Inoculation de la plante hôte et conditions de culture}

Les plants de trèfle ont été préparés de la même façon que l'orge. Des plants âgés d'une semaine 
Tableau II. Paramètres physico-chimiques du substrat utilisé.

\begin{tabular}{lc}
\hline Granulométrie $(\%)$ & \\
$2,00 \mathrm{~mm}-1,00 \mathrm{~mm}$ & 73,4 \\
$1,00 \mathrm{~mm}-0,50 \mathrm{~mm}$ & 25,6 \\
$0,50 \mathrm{~mm}-0,20 \mathrm{~mm}$ & 55,58 \\
$0,20 \mathrm{~mm}-0,10 \mathrm{~mm}$ & 9,06 \\
$0,10 \mathrm{~mm}-0,05 \mathrm{~mm}$ & 2,04 \\
$<0,05 \mathrm{~mm}$ & 0,34 \\
pH eau & 7,26 \\
Phosphore total $(\%)$ & 0,037 \\
Carbone organique $(\%)$ & 0,87 \\
Azote total $(\%)$ & 0,10 \\
Conductivité $(\mu \mathrm{s} / \mathrm{cm})$ & 143 \\
\hline
\end{tabular}

ont été repiqués dans des seaux contenant $4 \mathrm{~kg}$ du mélange tourbe-sable et à raison de 8 plantes par seau. L'inoculation par les champignons symbiotiques a été effectuée par un apport de 2,5 g (MF) de racines d'orge mycorhizées à proximité du système racinaire de chaque plante de trèfle. Les cultures de trèfle ont ensuite été réalisées dans un mélange sable-tourbe $(2: 1 \mathrm{v} / \mathrm{v})$ préalablement stérilisé pendant 3 heures à $180{ }^{\circ} \mathrm{C}$ [32] et dont les paramètres physico-chimiques sont donnés dans le tableau II.

La contrainte hydrique a été appliquée au moment du repiquage des plants inoculés de trèfle (une semaine après leur germination). Pour la fertilisation du sol, un apport hebdomadaire de $30 \mathrm{ml}$ de la solution nutritive Long Ashton modifiée [39] a été effectué pour tous les traitements. Les seaux ont ensuite été placés dans une serre en plastique transparent. Le suivi de l'évolution de la température et de l'humidité relative de l'air ambiant à l'intérieur de la serre (Tab. III) était effectué à l'aide d'un hygrothermographe (type Dickson, modèle THDX). La photopériode était de 16 heures.

Sept traitements fongiques [témoin non inoculé, Glomus (Aoufous), Sclérocystis (Aoufous), Glomus (Agdz), Sclérocystis (Agdz), complexe Aoufous et Glomus mosseae (souche de référence provenant de l'INRA de Dijon, France, Dr. Plenchette)] et deux traitements hydriques ( $75 \%$ et $30 \%$ de la capacité au champ) ont été utilisés. Pour chaque combinaison, 6 répétitions ont été traitées en randomisation.

\subsection{Paramètres mesurés}

Après deux mois de culture, des prélèvements de cinq plantes de trèfle par répétition et par traitement ont été effectués, pour évaluer l'effet de la contrainte hydrique sur la croissance et les caractéristiques hydriques des plantes mycorhizées et non mycorhizées ainsi que sur la croissance et le développement des champignons mycorhiziens.

Les racines des plantes de trèfle ont été traitées [38] et colorées au bleu trypan à $0.01 \%$ dans du lactoglycérol. L'examen de l'état de la mycorhization du système racinaire a été réalisé selon la méthode décrite par Trouvelot et al. [51] pour caractériser le développement et l'agressivité des champignons mycorhiziens en condition de contrainte hydrique.

La réponse des plantes de trèfle à la mycorhization a été estimée par la détermination du nombre de feuilles formées, la surface foliaire, l'allongement de la partie aérienne de la plante et la

Tableau III. Évolution des conditions climatiques à l'intérieur de la serre au cours de la culture des plantes de trèfle.

\begin{tabular}{|c|c|c|c|c|c|}
\hline Mois & $\begin{array}{c}\mathrm{T}^{\circ} \max \\
\text { moyenne }\left({ }^{\circ} \mathrm{C}\right)\end{array}$ & $\begin{array}{c}\mathrm{T}^{\circ} \min \\
\text { moyenne }\left({ }^{\circ} \mathrm{C}\right)\end{array}$ & $\begin{array}{c}\text { Paramètres mesurés } \\
\text { HR }(\%) \\
\text { moyenne nocturne }\end{array}$ & $\begin{array}{c}\text { HR }(\%) \\
\text { moyenne diurne }\end{array}$ & $\begin{array}{l}\text { Lumière } \\
\mu \mathrm{m}^{-2} \cdot \mathrm{s}^{-1}\end{array}$ \\
\hline Janvier & 29 & 11 & 97 & 56 & 330 \\
\hline Février & 28 & 12 & 96 & 56,25 & 330 \\
\hline
\end{tabular}


production de biomasse (matière sèche (MS) après séchage à l'étuve à $105^{\circ} \mathrm{C}$ pendant 24 heures) des échantillons racinaires et foliaires pris séparément.

L'accroissement (Acc) de la partie aérienne de la plante [39] a également été déterminé pour estimer l'apport de la symbiose mycorhizienne chez le trèfle soumis à une déficience en eau.

$$
\text { Acc. } \%=\frac{\mathrm{MS}_{\text {plante mycorhizée }}-\mathrm{MS}_{\text {plante non mycorhizée }}}{\mathrm{MS}_{\text {plante non mycorhizée }}} \times 100 .
$$

L'effet de la mycorhization sur la teneur en éléments minéraux chez le trèfle a été estimé par le dosage de $\mathrm{N}, \mathrm{P}, \mathrm{K}, \mathrm{Na}, \mathrm{Ca}$ et $\mathrm{Mg}$ dans les parties aériennes de la plante [24].

La teneur en eau (T.E.) a été déterminée par la différence entre la masse de matière fraîche (MF) et celle de matière sèche (MS), exprimée en gramme d'eau par gramme de MS (g/g MS).

Le déficit de saturation en eau (D.S.H.) a été mesuré sur la feuille de même rang pour toutes les plantes et pour tous les traitements. La masse fraîche (MF) de l'échantillon, constitué de 10 disques foliaires, a été déterminée juste après la récolte. La saturation des disques foliaires, a été réalisée par flottement sur de l'eau distillée pendant 24 heures à l'obscurité dans des boites de Pétri de $9 \mathrm{~cm}$ de diamètre. Les échantillons ont ensuite été récupérés, égouttés entre deux feuilles de papier filtre, puis pesés pour la détermination de la masse fraîche saturée (MF sat). La masse sèche (MS) a été déterminée après un séjour à l'étuve à $100{ }^{\circ} \mathrm{C}$, pendant 24 heures. Le déficit de saturation en eau a ainsi été défini par la relation suivante [52] :

$$
\text { D.S.H. } \%=\frac{\text { MFsat }- \text { MF }}{\text { MFsat }- \text { MS }} \times 100 \text {. }
$$

Le potentiel hydrique foliaire $(\psi)$ a été mesuré par la méthode de la chambre de pression mise au point par Scholander et al. [44]. Il correspond à la pression de l'air exercée sur la feuille et affichée par le manomètre de la chambre en acier, qui permet la sortie d'une petite goutte d'eau du pétiole.

Le potentiel osmotique $(\pi)$ a été mesuré sur la feuille entière de la plante à la huitième semaine de croissance. La feuille récoltée était rapidement mise à congeler pendant 24 heures. Ensuite elle a été enfermée dans une enceinte en acier connectée à un psychromètre de type HR-33T Microvoltmeter.

La résistance des stomates et la transpiration foliaire ont été déterminées sur des échantillons de même type que ceux prélevés pour la détermination du D.S.H., à l'aide d'un poromètre type LI1600 .

Tous les résultats ont été analysés statistiquement avec le logiciel STAT-ITCF. Cette analyse est réalisée sur l'interaction des différentes souches fongiques avec les deux niveaux hydriques imposés au sol. L'étude comprend une analyse de variance suivie du test de Newman et Keuls au seuil de $5 \%$.

\section{Résultats}

\subsection{Effet du stress hydrique sur la colonisation des racines du trèfle par les champignons mycorhiziens}

La fréquence d'infection $(F)$ du système racinaire du trèfle par les champignons mycorhiziens à arbuscules (MA) est affectée par le déficit hydrique du sol (Fig. 1A). En effet, le dessèchement du sol provoque une baisse de la fréquence d'infection des racines de trèfle. Pour les deux traitements hydriques, la fréquence de mycorhization est plus importante dans le cas de l'inoculation par Glomus mosseae et le complexe d'Aoufous que par les autres isolats fongiques. Les Glomus et Sclerocystis d'Aoufous ont montré des fréquences d'infection plus élevées par rapport à ceux d'Agdz. Après 8 semaines de culture, la réduction de la fréquence de mycorhization pour une contrainte hydrique de $30 \%$ de la CC est de $70 \%$ dans le cas de l'inoculation par Glomus d'Agdz et de $40 \%$ pour Glomus d'Aoufous. Nous notons cependant, que le Glomus mosseae est peu affecté par le dessèchement du sol ( $\mathrm{F}=80 \%$ à $30 \% \mathrm{CC})$. L'intensité de colonisation de la racine de trèfle par les champignons MA diminue avec le dessèchement 


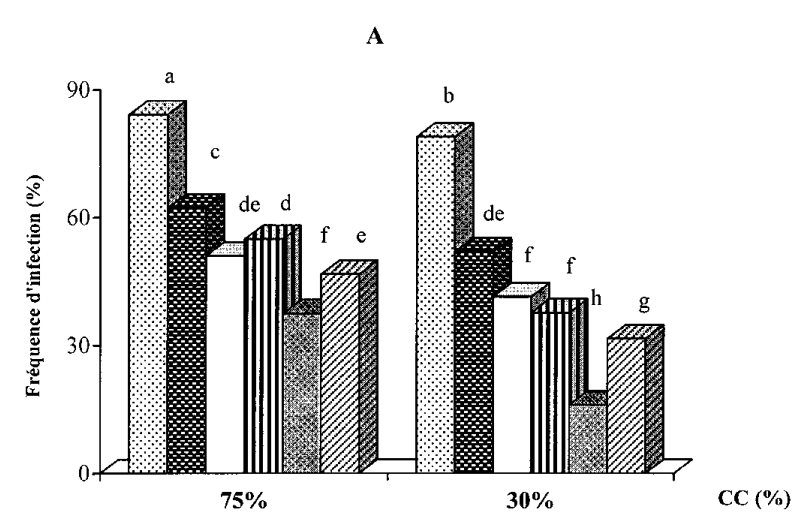

B

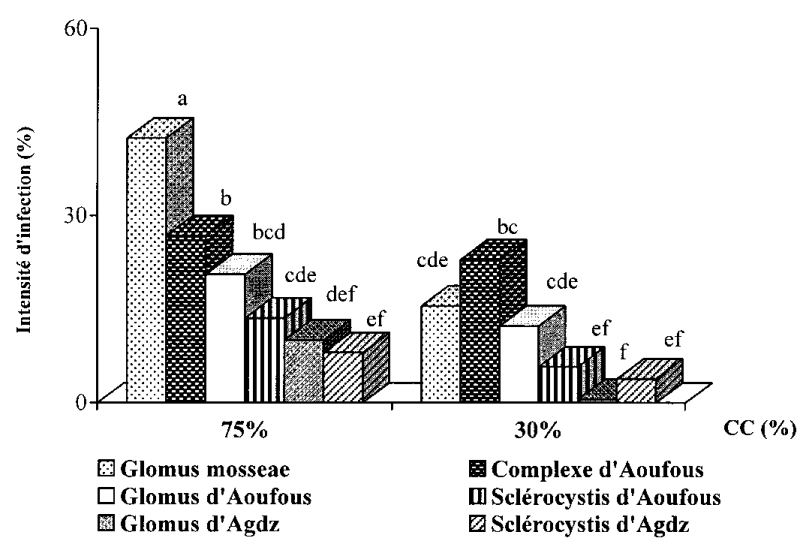

Figure 1: Effet de la contrainte hydrique sur la fréquence d'infection (A) et l'intensité de colonisation (B) des racines de trèfle, après 8 semaines de croissance. du sol (Fig. 1B). Les valeurs obtenues pour les genres Glomus et Sclérocystis isolés des sols d'Aoufous et d'Agdz restent relativement faibles par rapport à celles de Glomus mosseae et du complexe d'Aoufous. Comme le montre l'analyse statistique, l'application d'une contrainte hydrique de $30 \%$ de la CC réduit la progression des champignons mycorhiziens dans le cortex racinaire du trèfle. Glomus Agdz est le plus sensible au dessèchement en ayant le plus bas pourcentage de colonisation à $30 \% \mathrm{CC}$.

\subsection{Effet de la mycorhization sur la croissance du trèfle soumis à un déficit hydrique}

La surface foliaire du trèfle est augmentée de manière significative par la colonisation des différents champignons mycorhiziens et ce quel que soit le traitement hydrique imposé au sol (Tabl. IV). Elle est de $7 \mathrm{~cm}^{2}$ environ chez le trèfle inoculé par le Glomus d'Aoufous, le complexe d'Aoufous et le Glomus mosseae et elle n'est que de $2,5 \mathrm{~cm}^{2}$ chez le trèfle non mycorhizé pour un traitement hydrique de $75 \%$ de la capacité au champ. Pour un stress hydrique correspondant à $30 \%$ de la CC, on note une diminution de la surface foliaire. Seule la mycorhization due soit au Glomus d'Aoufous, au complexe d'Aoufous et au Glomus mosseae permet d'obtenir un développement foliaire significativement supérieur à celui du

Tableau IV. Effet de la contrainte hydrique sur les paramètres de croissance du trèfle non mycorhizé (témoin) et mycorhizé après 8 semaines de croissance.

\begin{tabular}{|c|c|c|c|c|c|c|}
\hline & \multicolumn{3}{|c|}{$75 \% \mathrm{CC}$} & \multicolumn{3}{|c|}{$30 \% \mathrm{CC}$} \\
\hline & $\mathrm{SF}\left(\mathrm{cm}^{2}\right)$ & NF & $\mathrm{HT}(\mathrm{cm})$ & $\mathrm{SF}\left(\mathrm{cm}^{2}\right)$ & $\mathrm{NF}$ & $\mathrm{HT}(\mathrm{cm})$ \\
\hline Glomus mosseae & $6,68 \mathrm{a}$ & $30,80 \mathrm{a}$ & $82,00 \mathrm{a}$ & $2,74 \mathrm{c}$ & $13,80 \mathrm{bc}$ & $50,00 \mathrm{c}$ \\
\hline Complexe d'Aoufous & $7,29 \mathrm{a}$ & $31,20 \mathrm{a}$ & $78,20 \mathrm{a}$ & $2,82 \mathrm{c}$ & $16,20 \mathrm{bc}$ & $45,40 \mathrm{~cd}$ \\
\hline Glomus d'Aoufous & $7,40 \mathrm{a}$ & $25,80 \mathrm{a}$ & $65,60 \mathrm{~b}$ & $3,04 \mathrm{c}$ & $12,80 \mathrm{bc}$ & $36,80 \mathrm{de}$ \\
\hline Sclerocystis d'Aoufous & $5,17 \mathrm{~b}$ & $18,20 \mathrm{~b}$ & $63,60 \mathrm{~b}$ & $2,17 \mathrm{c}$ & $13,40 \mathrm{bc}$ & $47,20 \mathrm{~cd}$ \\
\hline Glomus d'Agdz & $4,13 \mathrm{~b}$ & $18,60 \mathrm{~b}$ & $60,80 \mathrm{~b}$ & $2,13 \mathrm{c}$ & $8,80 \mathrm{bc}$ & 28,20 ef \\
\hline Sclerocystis d'Agdz & $4,95 \mathrm{~b}$ & $13,20 \mathrm{bc}$ & $62,20 \mathrm{~b}$ & $1,85 \mathrm{c}$ & $9,40 \mathrm{bc}$ & $43,60 \mathrm{~cd}$ \\
\hline Témoin & $2,43 \mathrm{c}$ & $13,00 \mathrm{bc}$ & $49,20 \mathrm{c}$ & $1,84 \mathrm{c}$ & $7,40 \mathrm{c}$ & $21,26 \mathrm{f}$ \\
\hline
\end{tabular}

SF : surface foliaire ; NF : nombre de feuilles ; HT : hauteur de la partie aérienne.

Les valeurs suivies de la même lettre ne sont pas significativement différentes $\mathrm{P}<0,05$ (test de Newman et Keuls). 
témoin. L'étude de la vitesse d'apparition des feuilles en fonction de la mycorhization et des traitements hydriques a montré que l'infection du trèfle par les champignons mycorhiziens d'Aoufous et de Glomus mosseae permet la formation d'un nombre de feuilles significativement supérieur à celui produit par les plantes non mycorhizées. De même que pour la surface foliaire, le nombre de feuilles formées diminue lorsque la réserve en eau disponible du sol diminue. Le Glomus d'Agdz n'a pas eu d'effet sur le nombre de feuilles formées chez le trèfle soumis à un stress de 30 \% CC. La mycorhization a eu aussi comme effet d'accroître la hauteur de la partie aérienne du trèfle à la huitième semaine de croissance. Lorsque la contrainte hydrique s'accentue, l'allongement de la partie aérienne du trèfle est réduit. Ces diminutions de l'allongement sont plus importantes chez le témoin que chez le trèfle mycorhizé et ce quel que soit le régime hydrique imposé. La mycorhization du trèfle par Glomus d'Agdz n'a pas eu d'effet sur la hauteur de la partie aérienne de la plante sévèrement stressée.

La production de matière sèche aérienne est accrue de manière significative par la colonisation du trèfle par les différents champignons mycorhiziens, excepté le Glomus et le Sclerocystis d'Agdz, que les conditions d'alimentation en eau soient favorables $(75 \% \mathrm{CC})$ ou limitantes $(30 \% \mathrm{CC})$ (Fig. 2). C'est ainsi que le Glomus mosseae permet une amélioration de la biomasse de $104 \%$ des plantes de trèfle soumises au régime hydrique de $75 \%$ de la capacité au champ.

Pour les deux traitements hydriques, le facteur accroissement obtenu lors de la mycorhization du trèfle par le Glomus mosseae, le complexe Aoufous, le Sclerocystis et le Glomus Aoufous est de $211 \%, 156 \%, 136 \%$ et $125 \%$ respectivement. Il varie entre $53 \%$ et $93 \%$ chez le trèfle colonisé par Glomus ou Sclerocystis d'Agdz (Fig. 3).

L'inoculation par les isolats du complexe Aoufous et le Glomus mosseae entraîne un accroissement significatif de la teneur en $\mathrm{P}$ des parties aériennes du trèfle, et ce, au niveau des deux traitements hydriques du sol (Tab. V). Dans les conditions favorables d'alimentation en eau $(75 \% \mathrm{CC})$,

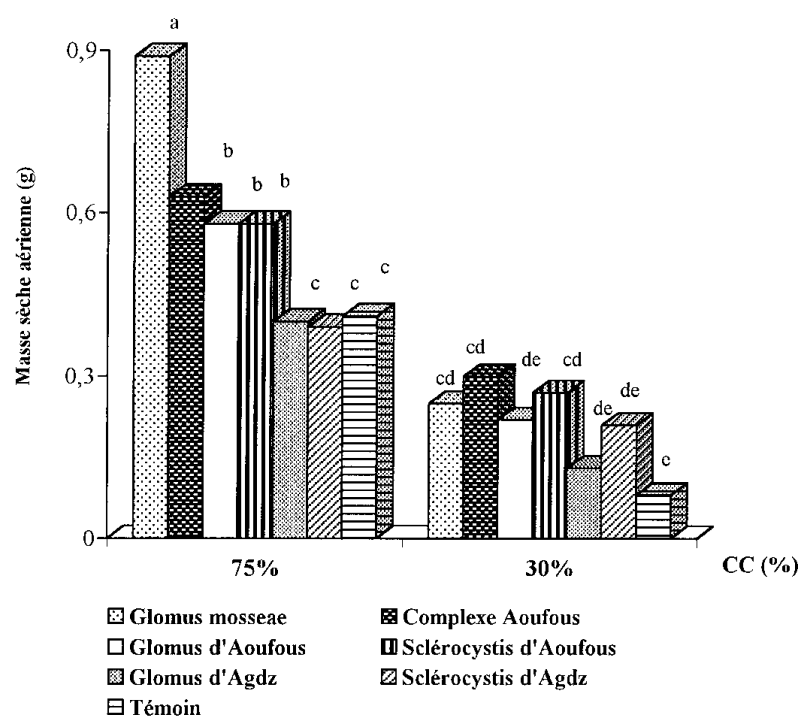

Figure 2 : Effet de la contrainte hydrique sur la production de matière sèche chez le trèfle témoin et mycorhizé, après 8 semaines.

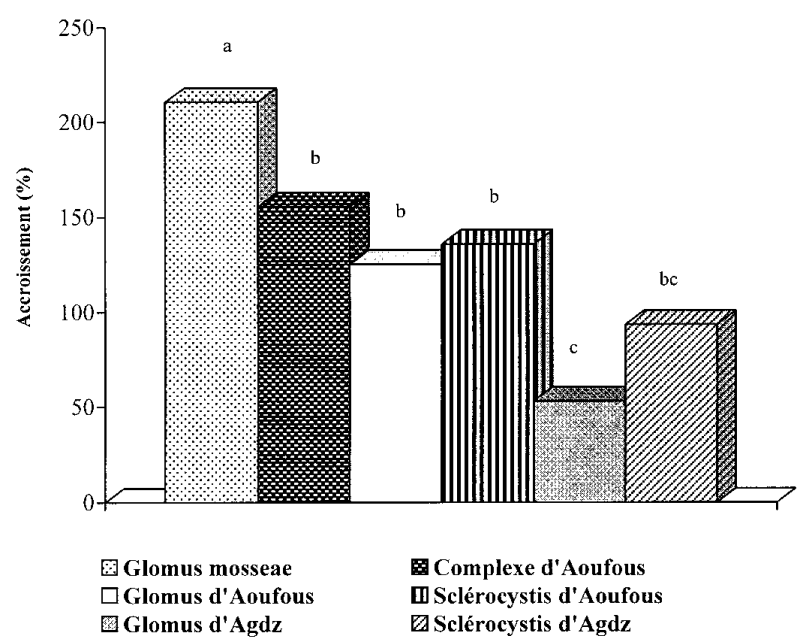

Figure 3 : Accroissement chez le trèfle mycorhizé par les différents isolats fongiques, après 8 semaines de croissance.

les plantes mycorhizées accumulent des teneurs en $\mathrm{N}$ plus élevées que les plantes non mycorhizées, cette tendance est inversée dans le cas de l'application d'une contrainte de $30 \%$ CC. Les teneurs en $\mathrm{K}, \mathrm{Mg}$ et en $\mathrm{Na}$ n'ont cependant pas été influencées par la mycorhization, quel que soit le régime hydrique imposé. 
Tableau V. Effet de la mycorhization et de la contrainte hydrique sur la teneur en éléments minéraux du trèfle.

\begin{tabular}{|c|c|c|c|c|c|c|c|}
\hline & Traitement & $\begin{array}{c}\mathrm{P} \\
\mathrm{mg} / \mathrm{g} \mathrm{MS}\end{array}$ & $\begin{array}{c}\mathrm{N} \\
\mathrm{mg} / \mathrm{g} \mathrm{MS}\end{array}$ & $\begin{array}{c}\mathrm{K} \\
\mathrm{mg} / \mathrm{g} \mathrm{MS}\end{array}$ & $\begin{array}{c}\mathrm{Ca}^{2+} \\
\mathrm{mg} / \mathrm{g} \mathrm{MS}\end{array}$ & $\begin{array}{c}\mathrm{Mg}^{2+} \\
\mathrm{mg} / \mathrm{g} \mathrm{MS}\end{array}$ & $\begin{array}{c}\mathrm{Na} \\
\mathrm{mg} / \mathrm{g} \mathrm{MS}\end{array}$ \\
\hline \multirow[t]{7}{*}{$75 \% \mathrm{CC}$} & Glomus mosseae & $6,50 \mathrm{a}$ & $9,50 \mathrm{~cd}$ & 24,90 & $7,30 \mathrm{~d}$ & 10,30 & 6,00 \\
\hline & Complexe d' Aoufous & $6,20 \mathrm{ab}$ & $9,50 \mathrm{~cd}$ & 24,70 & $11,20 \mathrm{bcd}$ & 10,90 & 4,00 \\
\hline & Glomus Aoufous & 4,90 cde & 7,70 de & 23,90 & $11,90 \mathrm{bc}$ & 9,70 & 3,70 \\
\hline & Glomus Agdz & $3,90 \mathrm{fg}$ & $9,10 \mathrm{~cd}$ & 24,20 & $9,80 \mathrm{bcd}$ & 9,40 & 3,30 \\
\hline & Sclerocystis Aoufous & 4,40 ef & $8,90 \mathrm{~cd}$ & 24,10 & $12,80 \mathrm{bc}$ & 10,80 & 4,00 \\
\hline & Sclerocystis Agdz & $5,80 \mathrm{abc}$ & $7,80 \mathrm{de}$ & 23,50 & $11,20 \mathrm{bcd}$ & 11,50 & 5,30 \\
\hline & Témoin & $5,40 \mathrm{bcd}$ & $6,10 \mathrm{e}$ & 22,90 & $12,60 \mathrm{bc}$ & 9,80 & 5,30 \\
\hline \multirow[t]{7}{*}{$30 \% \mathrm{CC}$} & Glomus mosseae & $5,50 \mathrm{bcd}$ & $13,00 \mathrm{ab}$ & 20,70 & $8,50 \mathrm{~cd}$ & 10,10 & 7,00 \\
\hline & Complexe d' Aoufous & $4,60 \mathrm{def}$ & $15,30 \mathrm{a}$ & 20,20 & $10,60 \mathrm{bcd}$ & 10,40 & 7,30 \\
\hline & Glomus d'Aoufous & $3,00 \mathrm{gh}$ & $13,90 \mathrm{a}$ & 23,90 & $14,20 \mathrm{ab}$ & 8,70 & 7,60 \\
\hline & Glomus d'Agdz & $3,10 \mathrm{gh}$ & $13,7 \mathrm{a}$ & 25,50 & $16,70 \mathrm{a}$ & 9,00 & 8,00 \\
\hline & Sclerocystis d'Aoufous & $3,70 \mathrm{fg}$ & $11,20 \mathrm{bc}$ & 22,50 & $13,00 \mathrm{bc}$ & 10,40 & 6,30 \\
\hline & Sclerocystis d'Agdz & $3,30 \mathrm{gh}$ & $10,70 \mathrm{bcd}$ & 18,70 & $12,40 \mathrm{bc}$ & 10,10 & 8,70 \\
\hline & Témoin & $2,60 \mathrm{~h}$ & $15,70 \mathrm{a}$ & 20,80 & $17,00 \mathrm{a}$ & 7,40 & 8,00 \\
\hline
\end{tabular}

Les valeurs suivies de la même lettre ne sont pas significativement différentes au seuil $\mathrm{P}<0,05$ à l'intérieur d'une même colonne et pour les deux CC imposés au sol (test de Newman et Keuls).

\subsection{Effet de la mycorhization et de la contrainte hydrique sur les paramètres hydriques du trèfle}

De façon générale, les plantes de trèfle mycorhizées maintiennent une importante teneur en eau au niveau de leur partie aérienne par rapport aux plantes témoins même en cas de stress hydrique sévère (Tab. VI). A $30 \%$ de la CC, les plantes inoculées par le Glomus mosseae ont montré une teneur en eau significativement supérieure à celle du témoin et qui est similaire à celle des plantes non mycorhizées et irriguées à $75 \%$ de la CC. Lors de l'application de la contrainte hydrique sévère de $30 \% \mathrm{CC}$, le déficit de saturation hydrique (D.S.H.) foliaire augmente chez les témoins et les plantes mycorhizées par Glomus ou Sclerocystis d'Aoufous et d'Agdz, tandis qu'il reste faible chez le trèfle inoculé avec les isolats du complexe Aoufous ou de Glomus mosseae (12 $13 \%$, à $30 \%$ de CC). Lorsque le déficit hydrique

Figure 4 : Effet de la contrainte hydrique sur le potentiel hydrique $(\mathrm{A})$ et osmotique $(\mathrm{B})$ foliaire chez le trèfle témoin et inoculé, après 8 semaines de croissance.

Les valeurs suivies de la même lettre ne sont pas significativement différentes; $(\mathrm{P}<0.05)$. CC : capacité au champ.

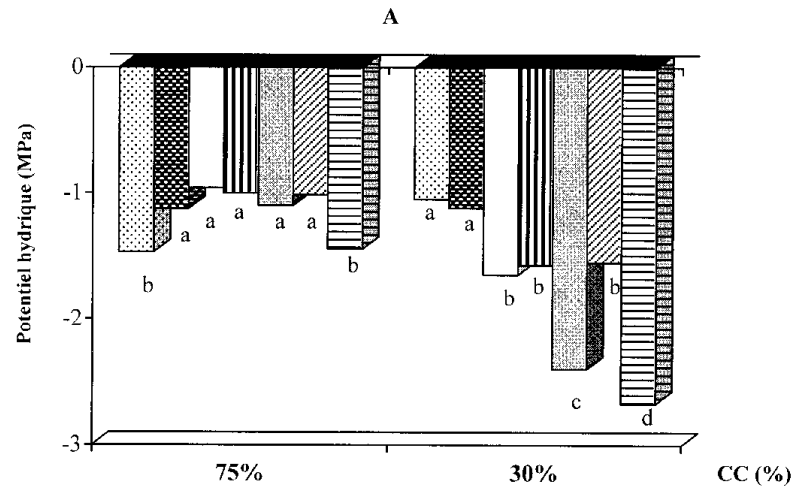

B

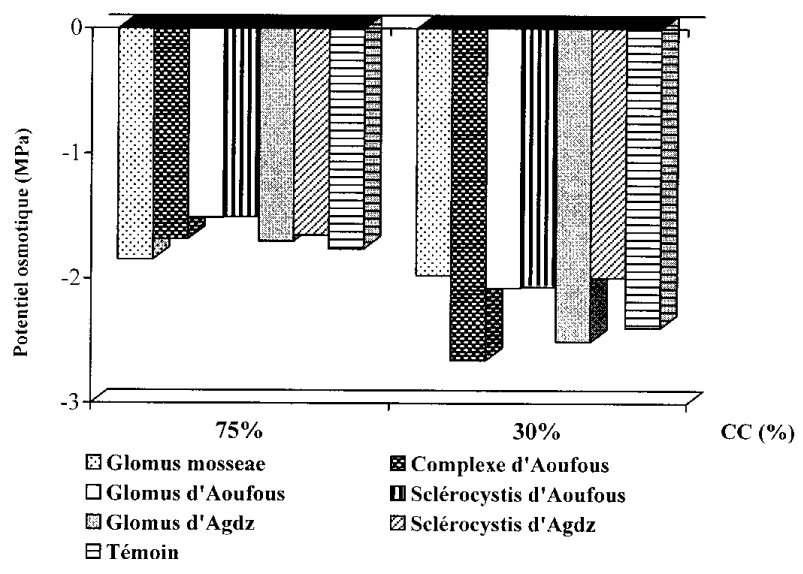


Tableau VI. Effet du stress hydrique et de la mycorhization sur les paramètres hydriques du trèfle, après 8 semaines de croissance.

\begin{tabular}{|c|c|c|c|}
\hline & Traitement & $75 \%$ CC & $30 \% \mathrm{CC}$ \\
\hline T.E (g/gMS) & $\begin{array}{l}\text { Glomus mosseae } \\
\text { Complexe d'Aoufous } \\
\text { Glomus d'Aoufous } \\
\text { Sclerocystis d'Aoufous } \\
\text { Glomus d'Agdz } \\
\text { Sclerocystis Agdz } \\
\text { Témoin }\end{array}$ & $\begin{array}{c}3,91 \mathrm{a} \\
4,18 \mathrm{a} \\
3,65 \mathrm{ab} \\
2,90 \mathrm{c} \\
3,44 \mathrm{~b} \\
3,53 \mathrm{~b} \\
3,04 \mathrm{c}\end{array}$ & $\begin{array}{c}2,91 \mathrm{c} \\
2,74 \mathrm{~cd} \\
2,40 \mathrm{de} \\
2,25 \mathrm{e} \\
1,69 \mathrm{f} \\
2,46 \mathrm{de} \\
1,90 \mathrm{f}\end{array}$ \\
\hline D.S.H (\%) & $\begin{array}{l}\text { Glomus mosseae } \\
\text { Complexe d'Aoufous } \\
\text { Glomus d'Aoufous } \\
\text { Sclerocystis d'Aoufous } \\
\text { Glomus d'Agdz } \\
\text { Sclerocystis d'Agdz } \\
\text { Témoin }\end{array}$ & $\begin{array}{c}15,04 \mathrm{~cd} \\
13,98 \mathrm{~d} \\
17,72 \mathrm{bcd} \\
16,68 \mathrm{bcd} \\
10,21 \mathrm{~d} \\
12,05 \mathrm{~d} \\
11,87 \mathrm{~d}\end{array}$ & $\begin{array}{c}12,34 \mathrm{~d} \\
13,01 \mathrm{~d} \\
22,20 \mathrm{abc} \\
26,83 \mathrm{a} \\
23,40 \mathrm{ab} \\
25,81 \mathrm{a} \\
28,32 \mathrm{a}\end{array}$ \\
\hline $\mathrm{R}(\mathrm{s} / \mathrm{cm})$ & $\begin{array}{l}\text { Glomus mosseae } \\
\text { Complexe d'Aoufous } \\
\text { Glomus d'Aoufous } \\
\text { Sclerocystis d'Aoufous } \\
\text { Glomus d'Agdz } \\
\text { Sclerocystis d'Agdz } \\
\text { Témoin }\end{array}$ & $\begin{array}{l}0,30 \mathrm{f} \\
0,25 \mathrm{f} \\
0,33 \mathrm{f} \\
0,33 \mathrm{f} \\
0,32 \mathrm{f} \\
0,31 \mathrm{f} \\
0,41 \text { ef }\end{array}$ & $\begin{array}{c}0,67 \mathrm{c} \\
0,62 \mathrm{~cd} \\
0,67 \mathrm{c} \\
0,45 \mathrm{def} \\
1,14 \mathrm{~b} \\
0,58 \mathrm{cde} \\
1,43 \mathrm{a}\end{array}$ \\
\hline $\mathrm{T}(\mathrm{cm} / \mathrm{s})$ & $\begin{array}{l}\text { Glomus mosseae } \\
\text { Complexe d'Aoufous } \\
\text { Glomus d'Aoufous } \\
\text { Sclerocystis d'Aoufous } \\
\text { Glomus d'Agdz } \\
\text { Sclerocystis d'Agdz } \\
\text { Témoin }\end{array}$ & $\begin{array}{c}37,76 \mathrm{~b} \\
56,13 \mathrm{a} \\
34,40 \mathrm{~b} \\
18,65 \mathrm{~cd} \\
25,65 \mathrm{c} \\
20,54 \mathrm{~cd} \\
39,38 \mathrm{~b}\end{array}$ & $\begin{array}{l}22,92 \mathrm{~cd} \\
25,70 \mathrm{c} \\
20,13 \mathrm{~cd} \\
17,65 \mathrm{~cd} \\
15,90 \mathrm{~cd} \\
15,37 \mathrm{~cd} \\
12,22 \mathrm{~d}\end{array}$ \\
\hline
\end{tabular}

T.E : teneur en eau ; D.S.H : déficit de saturation hydrique ; R : résistance des stomates ; T : transpiration foliaire.

Les valeurs suivies de la même lettre ne sont pas significativement différentes au seuil $\mathrm{P}<0,05$ et pour les deux CC imposés au sol (test de Newman et Keuls).

du sol s'accentue, la résistance des stomates augmente et la transpiration des feuilles diminue. Les plantes mycorhizées par les isolats du complexe Aoufous ou par Glomus mosseae présentent la transpiration foliaire la plus importante et la résistance des stomates la plus faible. C'est ainsi que les valeurs de la résistance des stomates sont deux fois plus importantes et les taux de transpiration foliaires sont deux fois moins élevés chez les plantes non mycorhizées que chez les plantes colonisées par le complexe d'Aoufous ou par le Glomus mosseae.
Lorsque le sol est soumis à une humidité équivalente à $30 \% \mathrm{CC}$, le potentiel hydrique foliaire diminue considérablement pour atteindre -2,7 $\mathrm{MPa}$ chez le trèfle témoin et -2,4 MPa chez le trèfle inoculé avec Glomus d'Agdz (Fig. 4A). Par contre, les plantes inoculées avec les autres isolats fongiques maintiennent leur potentiel hydrique à des valeurs élevées entre -0,8 $\mathrm{MPa}$ et -1,5 $\mathrm{MPa}$. Les plantes de trèfle mycorhizées par les isolats d'Aoufous ou par le Glomus mosseae, soumises à la contrainte de $30 \% \mathrm{CC}$, ont montré des potentiels hydriques significativement moins élevés que ceux des 
plantes non mycorhizées et irriguées à $75 \%$ de la capacité au champ. De même, le potentiel osmotique diminue avec le dessèchement du sol et se situe entre -2 et $-2,7 \mathrm{MPa}$ chez le trèfle mycorhizé et non mycorhizé, soumis à une contrainte hydrique de $30 \%$ CC (Fig. 4B). Pour les deux traitements hydriques imposés au sol, les variations du potentiel osmotique chez le trèfle entre les plants témoins et inoculés ne sont pas significatives.

\section{Discussion}

Le complexe Aoufous et le Glomus mosseae ont montré une meilleure aptitude à coloniser les racines du trèfle par rapport aux autres champignons mycorhiziens à arbuscules (MA). L'application d'une contrainte hydrique sévère induit une chute des paramètres de colonisation des champignons MA (fréquence et intensité de mycorhization). Le Glomus d'Agdz se propage faiblement dans le cortex racinaire du trèfle et se montre plus affecté par le dessèchement du sol que le Glomus ou le Sclérocystis d'Aoufous. Des réponses analogues ont été obtenues dans le cas des racines de laitue inoculées par Glomus occultum [42] et des plantes de blé mycorhizées [2] soumises à un déficit hydrique. La réduction de l'intensité des paramètres d'infectivité des champignons MA face à ce déficit hydrique peut être expliquée par les modifications physiologiques de la plante hôte [27]. En effet, la plupart de l'énergie utilisée pour le développement et la ramification des hyphes de champignons MA est obtenue des produits de la photosynthèse. Les diminutions des produits de photosynthèse de la plante pourraient affecter les statuts en hydrates de carbone de la racine, et par voie de conséquence le taux de colonisation des champignons MA [27, 48]. L'effet de la contrainte hydrique s'est manifesté différemment sur les associations champignons mycorhiziens-plantes de trèfle. Les plantes non mycorhizées se sont montrées plus sensibles aux variations du régime hydrique du sol que les plantes inoculées. Des résultats similaires ont été obtenus dans le cas du blé [3] et le soja [9] soumis à un stress hydrique appliqué au niveau du sol. L'inoculation par les isolats du complexe d'Aoufous et le Glomus mosseae permet une meilleure croissance des plantes de trèfle que l'inoculation par les autres champignons mycorhiziens. Ainsi la mycorhization par ces isolats fongiques a permis une nette amélioration du nombre de feuilles formées, de l'accroissement de la surface foliaire et de la hauteur de la plante, face à un déficit hydrique. De même, les productions en matière sèche sont améliorées par rapport aux plantes témoins. Des réponses analogues ont été signalées dans le cas de l'oignon et de la laitue inoculés avec le Glomus fasciculatum $[25,40,50]$ et le maïs inoculé avec le Glomus mosseae [28]. Ces résultats concordent avec nos résultats antérieurs $[1,35,36]$, étant donné que les isolats fongiques qui ont permis les meilleures croissance et nutrition minérale des plantes sont ceux qui ont présenté les meilleurs taux de colonisation racinaire. L'effet de la mycorhization quant aux modifications des paramètres hydriques du trèfle soumis à une contrainte hydrique est bien marqué. Ainsi la mycorhization par les différents champignons mycorhiziens induit des améliorations des paramètres hydriques de la plante. En effet, en présence d'une contrainte hydrique sévère ( 30 \% CC), la mycorhization du trèfle par les isolats d'Aoufous ou le Glomus mosseae permet à la plante de maintenir sa teneur en eau et son potentiel hydrique à des valeurs élevées au niveau de la partie aérienne de la plante. Des réponses analogues on été signalées dans le cas de plusieurs plantes mycorhizées $[14,16,18,22,33,34,41]$. Les plantes mycorhizées ont présenté de meilleurs taux de transpiration et des faibles résistances des stomates comparées aux plantes témoins. La faible résistance des stomates chez les plantes mycorhizées pourrait améliorer la fixation de $\mathrm{CO}_{2}$ au niveau du mésophylle [11]. L'amélioration de la fixation de $\mathrm{CO}_{2}$ contribue par voie de conséquence à une nette augmentation de la photosynthèse de la plante [29]. Aucune différence significative n'a été enregistrée au niveau du potentiel osmotique chez les plantes témoins et celles qui ont été inoculées. Ces résultats sont similaires à ceux obtenus par plusieurs auteurs $[6,12,17,18]$. Par contre, des plantes de blé inoculées avec le Glomus fasciculatum [3] ont présenté des chutes significatives de 
leur potentiel osmotique. Ces auteurs ont souligné le rôle des champignons MA, dans l'ajustement osmotique de la plante hôte. Les isolats d'Aoufous et le Glomus mosseae ont permis une augmentation $\mathrm{du}$ statut nutritionnel en $\mathrm{P}$ des plantes soumises à une déficience hydrique. Des effets similaires ont été notés chez les plantes mycorhizées et soumises à des conditions de stress hydrique [8, 19, 26, 49] et de stress salin [7, 43]. Les teneurs en $\mathrm{K}, \mathrm{Mg}$ et en $\mathrm{Na}$ ne sont cependant pas améliorées chez les plantes mycorhizées par rapport à celles non mycorhizées, ce qui pourrait être dû à la forte stimulation de la production de la matière sèche des trèfles mycorhizées. Cooper [13] et Blal [10] rapportent que les plantes mycorhizées peuvent contenir une quantité totale de ces éléments supérieure à celle des plantes non mycorhizées, mais vu leur croissance importante, donc leur biomasse, les concentrations en certains éléments y sont similaires ou plus faibles que celles des plantes non mycorhizées. Plusieurs auteurs ont expliqué que l'amélioration des paramètres hydriques chez les plantes mycorhizées et stressées par un manque d'eau est une conséquence de l'accroissement du statut nutritionnel et surtout de celui du phosphore $[5,20$, $30,34,37,53]$.

De cette étude, il ressort donc que les isolats d'Aoufous et le Glomus mosseae (souche de référence) sont plus agressifs et colonisateurs, même dans les conditions les plus limitantes en eau du sol (30 \% CC). Ces mêmes champignons ont permis une augmentation importante de la croissance, de la nutrition minérale et une amélioration des paramètres hydriques de la plante, quel que soit le régime hydrique imposé au sol. Par contre, Glomus d'Agdz n'a pas eu d'effet significatif sur la croissance et les contenus ioniques du trèfle stressé qui semble être plus affecté par le dessèchement du sol. Il est également intéressant de noter que les souches indigènes (complexe d'Aoufous) se sont montrées efficaces dans l'amélioration de la tolérance de la plante hôte au stress hydrique. De tels isolats fongiques autochtones et adaptés aux conditions défavorables pourraient constituer un moyen biologique efficace pour les plantes vivant continuellement sous stress hydrique accru.
Remerciements : Les auteurs remercient Dr. M. Honrubia, Professeur au département de botanique à l'Université de Murcia en Espagne et Dr. Charest de l'Université d'Ottawa, Canada, pour avoir lu et corrigé le manuscrit de cet article. Nous remercions également Pr. F. Marty de l'Université de Bourgogne, pour sa collaboration lors des prospections dans le sud marocain. Nous exprimons notre reconnaissance à la Fondation Internationale pour la Science (FIS) et le Conseil Régional de Bourgogne pour leurs soutiens financiers.

\section{Références}

[1] Abbas Y., Mycorhizes à arbuscules des zones arides : biodiversité et rôle dans la tolérance du trèfle (Trifolium alexandrinum) au stress salin, Thèse de $3^{\mathrm{e}}$ cycle, Faculté des Sciences Semlalia, Marrakech, 1998, $72 \mathrm{p}$.

[2] Al-karaki G.N., Al-raddad A., Effects of arbuscular mycorrhizal fungi and drought stress on growth and nutrient uptake of two wheat genotypes differing in drought resistance, Mycorrhiza 7 (1997) 83-88.

[3] Allen M.F., Boosalis M.G., Effects of two species of VA mycorrhizal fungi on drought tolerance of winter wheat, New Phytol. 93 (1983) 67-76.

[4] Al-raddad A., Mass production of Glomus mosseae spores, Mycorrhiza 5 (1995) 229-231.

[5] Andersen C.P., Markhart A.H., Dixon R.K., Root hydraulic conductivity of vesicular-arbuscular mycorrhizal green ash seedlings, New Phytol. 109 (1988) 465-471.

[6] Augé R.M., Stodola J.W., Ebel R.C., Duan X., Leaf elongation and water relations of mycorrhizal Sorghum in response to partial soil drying: two Glomus species at varying phosphorus fertilization, J. Exp. Bot. 46 (1995) 297-307.

[7] Azcon R., El-atrash F., Influence of arbuscular mycorrhizae and phosphorus fertilization on growth, nodulation and $\mathrm{N}_{2}$ fixation $\left({ }^{15} \mathrm{~N}\right)$ in Medicago sativa at four salinity levels, Biol. Fertil. Soils 24 (1997) 81-86.

[8] Barea J.M., Azcon C., Azcon R., Vesicular-arbuscular mycorrhizae improve both symbiotic $\mathrm{N}_{2}$ fixation and $\mathrm{N}$ uptake from soil as assessed with a ${ }^{15} \mathrm{~N}$ technique under field conditions, New Phytol. 106 (1987) $717-725$.

[9] Bethlenfalvay G.J., Brown M.S., Ames R.N., Thomas S., Effects of drought on host and endophyte 
development in mycorrhizal soybeans in relation to water use and phosphate uptake, Physiol. Plant. 72 (1988) 565-571.

[10] Blal B., Les endomycorhizes VA chez le palmier à huile (Elaeis guneesis jacq.) : rôle dans la régulation de la croissance et dans la nutrition minérale des jeunes plants de clones micropropagés, Thèse de Doctorat, Univ. Dijon, 1989, 93 p.

[11] Brown M.S., Bethelenfalvay G.J., Glycine Glomus-Rhizobium symbiosis, Plant Physiol. 85 (1987) 120-123.

[12] Bryla D.R., Duniway J.M., Growth, phosphorus uptake, and water relations of safflower and wheat infected with an arbuscular mycorrhizal fungus, New Phytol. 136 (1997) 581-590.

[13] Cooper K.M., Physiology of VA mycorrhizal associations, in: Powell C.L., Bagyraj Dj. (Eds.), VA mycorrhizae, CRC Press, Boca Raton, Florida, USA, 1984, pp. $155-186$.

[14] Dixon R.K., Rao M.V., Garg V.K., Water relations and gas exchange of mycorrhizal Leucaena leucocephalla seedlings, J. Trop. Forest Sci. 4 (1993) 542-552.

[15] Elachouri M., Effets du stress hydrique sur deux variétés de maïs, Diplôme d'étude approfondie, Faculté des Sciences de Tunis II, 1993, 89 p.

[16] Ellis J.R., Larsen H.J., Boosalis M.G., Drought resistance of wheat plants inoculated with vesiculararbuscular mycorrhizae, Plant and Soil 86 (1985) 369-378.

[17] Estaun M.V., Efecto de las micorrizas vesiculoarbusculares en las relaciones hidricas y en el crecimiento de plantas sometidas a estrés salino, Tesis Doctoral, Facultad de Ciencias Universidad de Granada, 1991, 139 p.

[18] Faber A., Zasoski R.J., Munns D., A method for measuring hyphal nutrient and water uptake in mycorrhizal plants, Can. J. Bot. 69 (1991) 87-94.

[19] Fitter A.H., Functioning of vesicular-arbuscular mycorrhizas under field conditions, New Phytol. 99 (1985) 257-265.

[20] Fitter A.H., Water relations of red clover Trifolium pratense $L$. as affected by VA mycorrhizal infection and phosphorus supply before and during drought, J. Exp. Bot. 39 (1988) 595-603.

[21] Gerdman J.W., Nicolson T.H., Spores of endogone species from soil by wet sieving and decanting, Trans. Br. Mycol. Soc. 46 (1963) 235-244.
[22] Graham J.H., Sylvertsen J.P., Influence of vesicular-arbuscular mycorrhiza on the hydraulic conductivity of roots of two Citrus rootstocks, New Phytol. 97 (1984) 277-284.

[23] Hall I.R., Taxonomy of VA mycorrhizal fungi, in: Powell C.L., Bagyraj Dj. (Eds.) VA mycorrhizae, CRC Press. Inc. Boca Raton, Florida, USA, 1984, pp. 57-94.

[24] Harvey R.M., Fox J.L., Nutrient removal using Lemna minor, J. Water Pollut. Control Fed. 9 (1973) 1928-1938.

[25] Hirrel M.C., Gerdemann J.W., Improved growth of onion and bell pepper in saline soils by two vesicular-arbuscular mycorrhizal fungi, Soil Sci. Soc. Am. J. 44 (1980) 654-655.

[26] Joner E.J., Jakobsen I., Uptake of ${ }^{32} \mathrm{P}$ from labelled organic matter by mycorrhizal and non-mycorrhizal subterranean clover (Trifolium subterraneum L.), Plant and Soil 172 (1995) 221-227.

[27] Juniper S., Abbott L., Vesicular-arbuscular mycorrhizas and soil salinity, Mycorrhiza 4 (1993) $45-57$.

[28] Kothari S.K., Marschner H., George E., Effect of VA mycorrhizal fungi and rhizosphere microorganisms on root and shoot morphology, growth and water relations in maize, New Phytol. 116 (1990) 303-311.

[29] Lawlor D.W., Stress metabolism: its implication in breeding programmes, in: Srivastava J.P., Porceddu E., Acevedo E., Varma S. Icarda (Eds.), Drought tolerance in winter cereals, John Wiley \& Sons Ltd, 1987, pp. 227-240.

[30] Lehto T., Mycorrhizas and drought resistance of Picea sitchensis (Bong) Carr. I. In conditions of nutrient deficiency, New Phytol. 122 (1992) 669-673.

[31] Mikola P., Mycorrhizae under tropical stresses, Angew. Bot. 61 (1987) 15-23.

[32] Montagne D., Saintmacary H., Méthode de culture semi-aseptique de légumineuses en pot, FAO/ GRET, 1983, pp. 1-3.

[33] Nelsen C.E., Safir G.R., Increased drought tolerance of mycorrhizal onion plants caused by improved phosphorus nutrition, Planta (1982) 407-413.

[34] Nelsen C.E., Safir G.R., The water relations of well-watered, mycorrhizal, and non-mycorrhizal onion plants, J. Am. Soc. Hort. Sci. 107 (1982) 271-274.

[35] Oihabi A., Étude de l'influence des endomycorhizes à vésicules et arbuscules sur le Bayoud et la nutrition du palmier dattier, Thèse de Doctorat d'État, Univ. Cadi Ayyad Marrakech, 1991, 117 p. 
[36] Oihabi A., Meddich A., Effet des mycorhizes à arbuscules sur la croissance et la composition minérale du trèfle (Trifolium alexandrinum), Cah. Agric. 5 (1996) 382-386.

[37] Ojala J.C., Jarrell W.M., Menge J.A., Johnson E.L.V., Influence of mycorrhizal fungi on the mineral nutrition and yield of onion in saline soil, Agron. J. 75 (1983) 255-259.

[38] Phillips J.M., Hayman D.S., Improved procedures for clearing roots and staining parasitic and vesicular-arbuscular mycorrhizal fungi for rapid assessment of infection, Trans. Brit. Mycol. Soc. 55 (1970) $158-161$.

[39] Plenchette C., Furlan V., Fortin J.A., Effects of different endomycorrhizal fungi on five host plants grown on calcined montmorillonite clay, J. Am. Soc. Hort. Sci. 107 (1982) 535-538.

[40] Ruiz-Lozano J.M., Azcon R., Hyphal contribution to water uptake in mycorrhizal plants as affected by the fungal species and water status, Physiol. Plant. 95 (1995) 472-478.

[41] Ruiz-Lozano J.M., Azcon R., Mycorrhizal colonization and drought stress as factors affecting nitrate reductase activity in lettuce plants, Agric. Ecosyst. \& Environ. 60 (1996) 175-181.

[42] Ruiz-Lozano J.M., Azcon R., Gomez M., Effect of arbuscular mycorrhizal Glomus species on drought tolerance: physiological and nutritional plant responses, Appl. Environ. Microbiol. 61 (1995) 456-460.

[43] Ruiz-Lozano J.M., Azcon R., Gomez M., Alleviation of salt stress by arbuscular-mycorrhizal Glomus species in Lactuca sativa plants, Physiol. Plant. 98 (1996) 767-772.

[44] Scholander P.F., Hammel H.T., Bradstreet E.D., Hemmingzen E.A., Sap pressure in vascular plants, Science 148 (1965) 339-346.

[45] Strullu D.G., Micropropagation of chesnut and conditions of mycorrhizal synthesis in vitro, New Phytol. 102 (1986) 95-101.
[46] Subramanian K.S., Charest C., Influence of arbuscular mycorrhizae on the metabolism of maize under drought stress, Mycorrhiza 5 (1995) 273-278.

[47] Subramanian K.S., Charest C., Nutritional, growth and reproductive responses of maize (Zea mays L.) to arbuscular mycorrhizal inoculation during and after drought stress at tasselling, Mycorrhiza 7 (1997) 25-32.

[48] Thomason B.D., Robson A.D., Abbott L.K., Mycorrhizas formed by Glomus fasciculatum on subterranean clover in Gigaspora calospora relation to soluble carbohydrate concentrations in roots, New Phytol. 114 (1990) 217-225.

[49] Tobar R., Azcon R., Barea J.M., Improved nitrogen uptake and transport from ${ }^{15} \mathrm{~N}$-labelled nitrate by external hyphae of arbuscular mycorrhiza under waterstressed conditions, New Phytol. 126 (1994) 119-122.

[50] Tobar R., Azcon R., Barea J.M., The improvement of plant $\mathrm{N}$ acquisition from an ammonium-treated, drought-stressed soil by the fungal symbiont in arbuscular mycorrhizae, Mycorrhiza 4 (1994) 105-108.

[51] Trouvelot A., Kouch J., Gianinazzi-Pearson V., Mesure du taux de mycorhization VA d'un système radiculaire : Recherche de méthodes d'estimation ayant une signification fonctionnelle, in: Gianinazzi S. (Ed.), Les mycorhizes : Physiologie et Génétique, $1{ }^{\text {er }}$ Séminaire Européen sur les mycorhizes, Dijon, INRA, Paris, 1986, pp. 217-221.

[52] Turner N.C., Techniques and experimental approaches for the measurements of plant water status, Plant and Soil 58 (1981) 339-366.

[53] Waterer D.R., Coltman R.R., Response of mycorrhizal bell peppers to inoculation timing, phosphorus, and water stress, Hortscience 4 (1989) 688-690.

[54] Weissenhorn I., Leyval C., Root colonization of maize by a Cd-sensitive and a Cd-tolerant Glomus mosseae and cadmium uptake in sand culture, Plant and Soil 175 (1995) 233-238. 